\title{
Blowup of Smooth Solutions for an Aggregation Equation
}

\author{
Wenxin $\mathrm{Yu}^{1}$ and Yigang $\mathrm{He}^{2}$ \\ ${ }^{1}$ College of Electrical and Information Engineering, Hunan University, Changsha, Hunan 410082, China \\ ${ }^{2}$ School of Electrical and Automation Engineering, Hefei University of Technology, Hefei, Anhui 230009, China
}

Correspondence should be addressed to Wenxin Yu; slowbird@sohu.com

Received 30 July 2013; Accepted 9 October 2013

Academic Editor: Changxing Miao

Copyright (C) 2013 W. Yu and Y. He. This is an open access article distributed under the Creative Commons Attribution License, which permits unrestricted use, distribution, and reproduction in any medium, provided the original work is properly cited.

We study the blowup criterion of smooth solutions for an inviscid aggregation equation in $\mathbb{R}^{n}$. By means of the losing estimates and the logarithmic Sobolev inequality, we establish an improved blowup criterion of smooth solutions.

\section{Introduction}

In this paper, we consider the following aggregation equation in $\mathbb{R}^{n}$ :

$$
\begin{gathered}
u_{t}+\nabla \cdot(u(\nabla K * u))=0, \\
u(x, 0)=u_{0}(x)
\end{gathered}
$$

with a given kernel $K: \mathbb{R}^{n} \rightarrow \mathbb{R}$. The unknown function $u$ is either the population density of a species or the density of particles in a granular medium. Aggregation equations of form (1) arise in many problems in biology, chemistry, and population dynamics and describe a collective motion and aggregation phenomena in biology and in mechanics of continuous media. From the mathematical point of view, (1) can be considered as a nonlinear, nonlocal transport equation, and its character depends strongly on properties of a given kernel $K$.

Laurent [1] has studied problem (1) in detail and proved several local and global existence results for a class of kernels $K$ with different regularity. Then, Bertozzi et al. [2-5] have proved finite-time blowup of solutions corresponding to compactly supported radial initial data. Those results can be summarized as follow. Kernels that are smooth (not singular) at origin $x=0$ lead to the global in time existence of solutions (see e.g., $[1,4]$ ). Nonsmooth kernels (and $C^{1}$ off the origin, like $K(x)=e^{-|x|}$ ) may lead to blowup of solutions either in finite or infinite time [1-4, 6, 7].

Equation (1) has been also intensively considered in the viscous case, namely, with the dissipative term $(-\Delta)^{\gamma} u$. The authors of [6-10] studied the problem (1) with fractional dissipation $(-\Delta)^{\gamma / 2} u$ and proved finite blowup of solutions or their global well-posedness for certain class of kernels. Recently, Karch and Suzuki [11] have classified kernels, which lead either to the blowup or global existence of solutions to (1) with the classical dissipation $\Delta u$.

Typical approaches to prove a finite-time aggregation include an extension of the method of characteristics $[4,12]$, the energy method (e.g., $[2,3,6,7]$ ) and the moment (or virial) method. The latter has been first applied to mean field models for self-gravitating particles and chemotaxis system [13] and recently in $[8,9,11]$.

Our aim in this paper is to present another method showing finite time blowup of a large class of solutions of (1). In the mixed time-space Besov spaces, using the losing estimates and the logarithmic Sobolev inequality, we can set up the blowup criterion at some $\Delta_{j}$ which is the frequency localization operator in the Littlewood-Paley decomposition. The blowup result we obtained for (1) had been proved under the assumptions much relaxed compared to $[6,7,12]$. In addition, it allows us to consider potentials which are more general than those considered in previous papers, namely, we require $\nabla K \in W^{1,1}\left(\mathbb{R}^{n}\right)$ which contains the case $K=e^{-|x|}$. Here, we follow the ideas introduced in $[2,14-18]$. Our main result reads as follows. 
Theorem 1. Let $\nabla K \in W^{1,1}\left(\mathbb{R}^{n}\right), u_{0} \in B_{p, q}^{s}, s>n / p+1,1<p$, $q<\infty$. Suppose that $u \in C\left([0, T) ; B_{p, q}^{s}\right) \cap C^{1}\left([0, T) ; B_{p, q}^{s-1}\right)$ is a smooth solution to (1). If there exists an absolute constant $M>0$ such that if

$$
\limsup _{\varepsilon \rightarrow 0} \int_{j \in \mathbb{Z}}^{T}\left\|\int_{T-\varepsilon} u\right\|_{\infty} d t=\delta<M,
$$

then $\delta=0$ and the solution $u$ can be extended past time $t=T$. In other words, if

$$
\limsup _{\varepsilon \rightarrow 0} \int_{j \in \mathbb{Z}}^{T}\left\|\int_{T-\varepsilon} u\right\|_{\infty} d t \geq M,
$$

then the solution blows up at $t=T$. Here, $\Delta_{j}$ is a frequency localization on $|\xi| \approx 2^{j}$; see Section 2 .

Note that $B_{p, q}^{s-1}$ is a Banach algebra for $s>n / p+1$. One can easily prove that there exists a unique smooth solution $u \in$ $C\left([0, T) ; B_{p, q}^{s}\right) \cap C^{1}\left([0, T) ; B_{p, q}^{s-1}\right)$ to (1) by standard method; see [19] for details.

Notation. Throughout the paper, $C$ stands for a generic constant. We will use the notation $A \lesssim B$ to denote the relation $A \leq C B$ and the notation $A \approx B$ to denote the relations $A \lesssim B$ and $B \lesssim A$.

\section{Preliminaries}

In this preparatory section, we provide the definition of some function spaces based on the so-called Littlewood-Paley decomposition and we review some important lemmas that will be used constantly in the sequel.

We start with the dyadic decomposition. Let $\varphi \in C_{0}^{\infty}\left(\mathbb{R}^{n}\right)$ be supported in the ring $\mathscr{C}:=\left\{\xi \in \mathbb{R}^{n}, 3 / 4 \leq|\xi| \leq 8 / 3\right\}$ and such that

$$
\sum_{q \in \mathbb{Z}} \varphi\left(2^{-q} \xi\right)=1 \quad \text { for } \xi \neq 0
$$

We define also the function $\chi(\xi)=1-\sum_{q \in \mathbb{N}} \varphi\left(2^{-q} \xi\right)$. Now for $u \in \mathcal{S}^{\prime}$ we set

$$
\begin{gathered}
\Delta_{-1} u=\chi(D) u ; \\
\forall q \in \mathbb{N}, \quad \Delta_{q} u=\varphi\left(2^{-q} D\right) u, \\
\forall q \in \mathbb{Z}, \quad \dot{\Delta}_{q} u=\varphi\left(2^{-q} D\right) u .
\end{gathered}
$$

The following low-frequency cut-off will be also used:

$$
\begin{gathered}
S_{q} u=\sum_{-1 \leq j \leq q-1} \Delta_{j} u, \\
\dot{S}_{q} u=\sum_{j \leq q-1} \dot{\Delta}_{j} u .
\end{gathered}
$$

Let us now recall the definition of Besov spaces through dyadic decomposition.
Let $(p, q) \in[1,+\infty]^{2}$ and $s \in \mathbb{R}$; then the inhomogeneous space $B_{p, q}^{s}$ is the set of tempered distributions $u$ such that

$$
\|u\|_{B_{p, q}^{s}}:=\left(2^{q s}\left\|\Delta_{q} u\right\|_{L^{p}}\right)_{\ell^{q}}<\infty .
$$

To define the homogeneous Besov spaces we first denote by $S^{\prime} / \mathscr{P}$ the space of tempered distributions modulo polynomials. Thus, we define the space $\dot{B}_{p, r}^{s}$ as the set of distribution $u \in \mathcal{S}^{\prime} / \mathscr{P}$ such that

$$
\|u\|_{\dot{B}_{p, q}^{s}}:=\left(2^{q s}\left\|\dot{\Delta}_{q} u\right\|_{L^{p}}\right)_{\ell^{q}}<\infty
$$

We point out that if $s>0$, then we have $B_{p, q}^{s}=\dot{B}_{p, q}^{s} \cap L^{p}$ and

$$
\|u\|_{B_{p, q}^{s}} \approx\|u\|_{\dot{B}_{p, q}^{s}}+\|u\|_{L^{p}} .
$$

In our next study, we require two kinds of coupled spacetime Besov spaces. The first one is defined in the following manner: for $T>0$ and $q \geq 1$, we denote by $L_{T}^{r} \dot{B}_{p, q}^{s}$ the set of all tempered distributions $u$ satisfying

$$
\|u\|_{L_{T}^{r} \dot{B}_{p, r}^{s}}:=\left\|\left(2^{q s}\left\|\dot{\Delta}_{q} u\right\|_{L^{p}}\right)_{\ell^{q}}\right\|_{L_{T}^{r}}<\infty
$$

The second mixed space is $\widetilde{L}_{T}^{r} \dot{B}_{p, q}^{s}$ which is the set of tempered distribution $u$ satisfying

$$
\|u\|_{\widetilde{L}_{T}^{r} \dot{B}_{p, q}^{s}}:=\left(2^{q s}\left\|\dot{\Delta}_{q} u\right\|_{L_{T}^{r} L^{p}}\right)_{\ell^{q}}<\infty .
$$

We can define by the same way the spaces $L_{T}^{r} B_{p, q}^{\mathcal{s}}$ and $\widetilde{L}_{T}^{r} B_{p, q}^{\mathcal{s}}$.

The following embeddings are a direct consequence of Minkowski's inequality.

Let $s \in \mathbb{R}, r \geq 1$, and $(p, q) \in[1, \infty]^{2}$; then we have

$$
\begin{gathered}
L_{T}^{r} \dot{B}_{p, q}^{s} \hookrightarrow \widetilde{L}_{T}^{r} \dot{B}_{p, q}^{s}, \quad \text { if } q \geq r, \\
\widetilde{L}_{T}^{r} \dot{B}_{p, q}^{s} \hookrightarrow L_{T}^{r} \dot{B}_{p, q}^{s}, \quad \text { if } r \geq q .
\end{gathered}
$$

Now we give two useful lemmas.

Lemma 2 (Bernstein's inequalities [20]). Let $1 \leq p \leq q \leq \infty$. Assume that $f \in L^{p}$; then there exists a constant $C$ independent of $f$, $j$ such that

$$
\begin{aligned}
\operatorname{supp} \hat{f} & \subset\left\{|\xi| \leq C 2^{j}\right\} \\
& \Longrightarrow\left\|\partial^{\alpha} f\right\|_{L^{q}} \leq C 2^{j|\alpha|+j n((1 / p)-(1 / q))}\|f\|_{L^{p}}, \\
\operatorname{supp} \hat{f} & \subset\left\{\frac{1}{C} 2^{j} \leq|\xi| \leq C 2^{j}\right\} \\
& \Longrightarrow\|f\|_{L^{p}} \leq C 2^{-j|\alpha|} \sup _{|\beta|=|\alpha|}\left\|\partial^{\beta} f\right\|_{L^{p}} .
\end{aligned}
$$


Lemma 3 (logarithmic Sobolev inequality). Let $1 \leq p<\infty$, $1 \leq q<\infty$, and $s>n / p+1$. Assume that $f \in \widetilde{L}_{T}^{1}\left(\dot{B}_{\infty, \infty}^{0}\right) \cap$ $L_{T}^{\infty}\left(B_{p, q}^{s-1}\right)$. Then, the following inequality holds:

$$
\begin{aligned}
& \int_{0}^{T}\|f(t)\|_{\infty} d t \\
& \quad \leq C\left(1+\sup _{j} \int_{0}^{T}\left\|\Delta_{j} f\right\|_{\infty} d t\left(1+\log ^{+}\left(T\|f\|_{L_{T}^{\infty}\left(B_{p, q}^{s-1}\right)}\right)\right)\right),
\end{aligned}
$$

where $\log ^{+} x=\log x$, for $x>1, \log ^{+} x=0$, for $x \leq 1$, and $C$ is an absolute constant independent of $f, T$.

The proof is rather standard and can be found in [14].

\section{Proof of Theorem 1}

Applying $\dot{\Delta}_{j}$ to (1), we have

$$
\partial_{t} \dot{\Delta}_{j} u+\nabla \cdot \dot{\Delta}_{j}(u(\nabla K * u))=0 .
$$

Multiplying (15) by $\left|\dot{\Delta}_{j} u\right|^{p-2} \dot{\Delta}_{j} u$ and integrating the obtained equation in $\mathbb{R}^{n}$ with respect to the space variable give

$$
\frac{1}{p} \frac{d}{d t}\left\|\dot{\Delta}_{j} u\right\|_{p}^{p}=-\int_{\mathbb{R}^{n}} \nabla \cdot \dot{\Delta}_{j}(u(\nabla K * u))\left|\dot{\Delta}_{j} u\right|^{p-2} \dot{\Delta}_{j} u d x .
$$

Let us now turn to estimate the right-hand term of the previous equation by Bony's decomposition [21]. We decompose $u(\nabla K * u)$ as a paraproduct

$$
\begin{aligned}
u(\nabla K * u)= & T_{u} \nabla K * u+T_{\nabla K * u} u+R(u, \nabla K * u) \\
= & \sum_{k} \dot{S}_{k-1} u \dot{\Delta}_{k}(\nabla K * u)+\sum_{k} \dot{S}_{k-1}(\nabla K * u) \dot{\Delta}_{k} u \\
& +\sum_{\left|k-k^{\prime}\right| \leq 1} \dot{\Delta}_{k} u \dot{\Delta}_{k^{\prime}}(\nabla K * u) \\
= & I+I I+I I I .
\end{aligned}
$$

For $I$, integrating by parts together with the Hölder inequality yields

$$
\begin{aligned}
& \left.\left|\int_{\mathbb{R}^{n}} \nabla \cdot \dot{\Delta}_{j}(I)\right| \dot{\Delta}_{j} u\right|^{p-2} \dot{\Delta}_{j} u d x \mid \\
& \leq \sum_{|k-j| \leq 4}\left\|\dot{S}_{k-1} u\right\|_{L^{\infty}}\left\|\dot{\Delta}_{k}(\nabla K * u)\right\|_{L^{p}}\left\|\dot{\Delta}_{j} u\right\|_{L^{p}}^{p-2}\left\|\nabla \dot{\Delta}_{j} u\right\|_{L^{p}} \\
& \quad \leq \sum_{|k-j| \leq 4} 2^{j-k}\left\|\dot{S}_{k-1} u\right\|_{L^{\infty}}\left\|\dot{\Delta}_{k}\left(\nabla^{2} K * u\right)\right\|_{L^{p}} \\
& \quad \times\left\|\dot{\Delta}_{j} u\right\|_{L^{p}}^{p-2}\left\|\dot{\Delta}_{j} u\right\|_{L^{p}} \\
& \quad \sum_{|k-j| \leq 4}\left\|\dot{S}_{k-1} u\right\|_{L^{\infty}}\left\|\dot{\Delta}_{k} u\right\|_{L^{p}}\left\|\dot{\Delta}_{j} u\right\|_{L^{p}}^{p-1} .
\end{aligned}
$$

We have similar estimates for $I I$ using twice integration by parts:

$$
\begin{aligned}
& \left.\left|\int_{\mathbb{R}^{n}} \nabla \cdot \dot{\Delta}_{j}(I I)\right| \dot{\Delta}_{j} u\right|^{p-2} \dot{\Delta}_{j} u d x \mid \\
& \quad \leq\left.\sum_{|k-j| \leq 4}\left|\int_{\mathbb{R}^{n}} \dot{S}_{k-1}(\nabla K * u) \dot{\Delta}_{k} u\right| \dot{\Delta}_{j} u\right|^{p-2} \nabla \dot{\Delta}_{j} u d x \mid \\
& \quad \leq\left.\sum_{|k-j| \leq 4}\left|\int_{\mathbb{R}^{n}} \dot{S}_{k-1}(\nabla K * u) \nabla\right| \dot{\Delta}_{j} u\right|^{p} d x \mid \\
& \quad \leq\left.\sum_{|k-j| \leq 4}\left|\int_{\mathbb{R}^{n}} \dot{S}_{k-1}(\Delta K * u)\right| \dot{\Delta}_{j} u\right|^{p} d x \mid \\
& \quad \leq \sum_{|k-j| \leq 4}\left\|\dot{S}_{k-1} u\right\|_{L^{\infty}}\left\|\dot{\Delta}_{j} u\right\|_{L^{p}}^{p} .
\end{aligned}
$$

For $I I I$, we have

$$
\begin{aligned}
& \left.\left|\int_{\mathbb{R}^{n}} \nabla \cdot \dot{\Delta}_{j}(I I I)\right| \dot{\Delta}_{j} u\right|^{p-2} \dot{\Delta}_{j} u d x \mid \\
& \quad \leq \sum_{\substack{\left|k-k^{\prime}\right| \leq 1 \\
k \geq j-3}}\left\|\dot{\Delta}_{k} u\right\|_{L^{\infty}}\left\|\dot{\Delta}_{k^{\prime}}(\nabla K * u)\right\|_{L^{p}} \\
& \quad \times\left\|\dot{\Delta}_{j} u\right\|_{L^{p}}^{p-2}\left\|\nabla \dot{\Delta}_{j} u\right\|_{L^{p}} \\
& \quad \leq \sum_{\substack{\left|k-k^{\prime}\right| \leq 1 \\
k \geq j-3}} 2^{j-k}\left\|\dot{\Delta}_{k} u\right\|_{L^{\infty}}\left\|\dot{\Delta}_{k^{\prime}}\left(\nabla^{2} K * u\right)\right\|_{L^{p}}\left\|\dot{\Delta}_{j} u\right\|_{L^{p}}^{p-1} \\
& \quad \leq \sum_{k \geq j-3}\left\|\dot{\Delta}_{k} u\right\|_{L^{\infty}}\left\|\dot{\Delta}_{k} u\right\|_{L^{p}}\left\|\dot{\Delta}_{j} u\right\|_{L^{p}}^{p-1} .
\end{aligned}
$$

Adding (18)-(20), we infer that

$$
\begin{aligned}
\frac{1}{p} \frac{d}{d t}\left\|\dot{\Delta}_{j} u\right\|_{L^{p}}^{p} \leq & \sum_{j \in \mathbb{Z}}\left\|\dot{S}_{k-1} u\right\|_{L^{\infty}}\left\|\dot{\Delta}_{j} u\right\|_{L^{p}}^{p} \\
& +\sum_{k \geq j-3}\left\|\dot{\Delta}_{k} u\right\|_{L^{\infty}}\left\|\dot{\Delta}_{k} u\right\|_{L^{p}}\left\|\dot{\Delta}_{j} u\right\|_{L^{p}}^{p-1} \\
\leq & \sum_{j \in \mathbb{Z}}\|u\|_{L^{\infty}}\left\|\dot{\Delta}_{j} u\right\|_{L^{p}}^{p} \\
& +\sum_{k \geq j-3}\|u\|_{L^{\infty}}\left\|\dot{\Delta}_{k} u\right\|_{L^{p}}\left\|\dot{\Delta}_{j} u\right\|_{L^{p}}^{p-1}
\end{aligned}
$$

where we use the inequalities $\left\|\dot{S}_{k-1} u\right\|_{L^{\infty}} \leq\|u\|_{L^{\infty}}$ and $\left\|\dot{\Delta}_{k} u\right\|_{L^{\infty}} \leq\|u\|_{L^{\infty}}$. Thus, we deduce

$$
\frac{d}{d t}\left\|\dot{\Delta}_{j} u\right\|_{p} \leqslant \sum_{j \in \mathbb{Z}}\|u\|_{L^{\infty}}\left\|\dot{\Delta}_{j} u\right\|_{L^{p}}+\sum_{k \geq j-3}\|u\|_{L^{\infty}}\left\|\dot{\Delta}_{k} u\right\|_{L^{p}},
$$


which implies that

$$
\begin{aligned}
\frac{d}{d t}\left\|\dot{\Delta}_{j} u\right\|_{L^{p}}^{q} \leq & q \sum_{j \in \mathbb{Z}}\|u\|_{L^{\infty}}\left\|\dot{\Delta}_{j} u\right\|_{L^{p}}^{q} \\
& +q \sum_{k \geq j-3}\|u\|_{L^{\infty}}\left\|\dot{\Delta}_{k} u\right\|_{L^{p}}\left\|\dot{\Delta}_{j} u\right\|_{L^{p}}^{q-1} .
\end{aligned}
$$

Set

$$
\Psi_{\lambda}\left(t^{\prime}, t\right)=\lambda \int_{t}^{t^{\prime}}\left\|u\left(t^{\prime \prime}\right)\right\|_{\infty} d t^{\prime \prime}, \quad \Psi_{\lambda}(t)=\Psi_{\lambda}(0, t) .
$$

Integrating (23) over $[0, t)$ with respect to time variable $\tau$ and then multiplying by $2^{q\left(j s-\Psi_{\lambda}(t)\right)}$ the both obtained inequality, we get

$$
\begin{aligned}
& 2^{q\left(j s-\Psi_{\lambda}(t)\right)}\left\|\dot{\Delta}_{j} u\right\|_{L^{p}}^{q}(t) \\
& \leq 2^{j q s}\left\|\dot{\Delta}_{j} u\right\|_{L^{p}}^{q}(0) \\
& +\int_{0}^{t} 2^{-q \Psi_{\lambda}(\tau, t)}\|u\|_{L^{\infty}}(\tau) \\
& \quad \times\left(\sum_{j \in \mathbb{Z}} 2^{q\left(j s-\Psi_{\lambda}(\tau)\right)}\left\|\Delta_{j} u\right\|_{L^{p}}^{q}\right. \\
& +\sum_{k \geq j-3} 2^{(j-k) s} 2^{(q-1)\left(j s-\Psi_{\lambda}(\tau)\right)} \\
& \left.\times\left\|\dot{\Delta}_{j} u\right\|_{L^{p}}^{q-1} 2^{k s-\Psi_{\lambda}(\tau)}\left\|\dot{\Delta}_{k} u\right\|_{L^{p}}\right) d \tau .
\end{aligned}
$$

Let

$$
\alpha_{j, T}=\sup _{t \in[0, T)} 2^{j s-\Psi_{\lambda}(t)}\left\|\dot{\Delta}_{j} u\right\|_{p}, \quad \alpha_{T}=\left\|\alpha_{j, T}\right\|_{l^{q}} .
$$

Taking the supremum over $[0, T)$ on both sides of inequality (25), we deduce that

$$
\begin{aligned}
\alpha_{j, T}^{q} \lesssim & \alpha_{j, 0}^{q}+\sup _{t \in[0, T)} \int_{0}^{t} 2^{-q \Psi_{\lambda}(\tau, t)}\|u\|_{L^{\infty}} d \tau \\
& \times\left(\alpha_{j, T}^{q-1} \sum_{j \in \mathbb{Z}} \alpha_{k, T}+\alpha_{j, T}^{q-1} \sum_{k \geq j-3} 2^{(j-k) s} \alpha_{k, T}\right) .
\end{aligned}
$$

By the definition of $\Psi_{\lambda}(\tau, t)$, we know

$$
\frac{d}{d \tau} 2^{-q \Psi_{\lambda}(\tau, t)}=q \lambda \log 2 \cdot 2^{-q \Psi_{\lambda}(\tau, t)}\|u\|_{\infty}(\tau) ;
$$

then we have

$$
\begin{aligned}
\int_{0}^{t} 2^{-q \Psi_{\lambda}(\tau, t)}\|u\|_{\infty} d \tau & =\int_{0}^{t} \frac{1}{q \lambda \log 2} \frac{d}{d \tau} 2^{-q \Psi_{\lambda}(\tau, t)} \\
& =\frac{1}{q \lambda \log 2}\left(1-2^{-q \Psi_{\lambda}(0, t)}\right) \\
& \leq \frac{1}{q \lambda \log 2}
\end{aligned}
$$

Taking the sum over $j$ of (27) then using (29) and the Young inequality lead to

$$
\alpha_{T}^{q} \lesssim\left\|u_{0}\right\|_{\dot{B}_{p, q}^{s}}^{q}+\frac{1}{q \lambda \log 2} \alpha_{T}^{q}
$$

Now if we choose $\lambda$ large enough such that

$$
\lambda>\frac{2}{q \log 2}
$$

then

$$
\alpha_{T} \lesssim\left\|u_{0}\right\|_{\dot{B}_{p, q}^{s}}^{q} .
$$

Next we estimate $\|u\|_{p}$. It is easy to obtain that

$$
\|u(t)\|_{L^{p}} \lesssim\left\|u_{0}\right\|_{L^{p}}+\int_{0}^{t}\|u(\tau)\|_{L^{\infty}}\|u(\tau)\|_{L^{p}} d \tau .
$$

Multiplying by $2^{-\Psi_{\lambda}(t)}$, both sides of the inequality yields

$$
\begin{aligned}
2^{-\Psi_{\lambda}(t)}\|u(t)\|_{L^{p}} \lesssim & \left\|u_{0}\right\|_{L^{p}} \\
& +\int_{0}^{t} 2^{-\Psi_{\lambda}(\tau, t)}\|u(\tau)\|_{L^{\infty}} 2^{-\Psi_{\lambda}(\tau)}\|u(\tau)\|_{L^{p}} d \tau,
\end{aligned}
$$

from which and (29) we have

$$
\begin{aligned}
& \sup _{t \in[0, T)} 2^{-\Psi_{\lambda}(t)}\|u(t)\|_{L^{p}} \\
& \quad \leq\left\|u_{0}\right\|_{L^{p}}+\frac{1}{q \lambda \log 2} \sup _{t \in[0, T)} 2^{-\Psi_{\lambda}(t)}\|u(t)\|_{L^{p}} .
\end{aligned}
$$

If $\lambda \gtrsim 2 / \log 2$, then

$$
\sup _{t \in[0, T)} 2^{-\Psi_{\lambda}(t)}\|u(t)\|_{p} \lesssim\left\|u_{0}\right\|_{p}
$$

Let us define

$$
\beta_{T}=\sup \left(\alpha_{T}, \sup _{t \in[0, T)} 2^{-\Psi_{\lambda}(t)}\|u(t)\|_{p}\right) .
$$

This together with (32) and (36) implies that

$$
\beta_{T} \lesssim\left\|u_{0}\right\|_{B_{p, q}^{s}} .
$$

In particular, we have

$$
\|u(t)\|_{B_{p, q}^{s}} \lesssim 2^{\Psi_{\lambda}(t)}\left\|u_{0}\right\|_{B_{p, q}^{s}}, \quad \forall t \in[0, T) .
$$

Applying Lemma 3 with $f(t)=u(t)$ and the embedding $B_{p, q}^{s} \hookrightarrow B_{p, q}^{s-1}$, we have

$$
\begin{aligned}
\int_{0}^{t}\|u\|_{L^{\infty}} d \tau \leqslant & 1+\sup _{j} \int_{0}^{t}\left\|\Delta_{j} u\right\|_{L^{\infty}} d \tau \\
& \times\left(1+\log ^{+}\left(t\|u\|_{L_{t}^{\infty}\left(B_{p, q}^{s}\right)}\right)\right) .
\end{aligned}
$$


For the sake of convenience, we denote

$$
\zeta(T)=\sup _{[0, T)}\|u(t)\|_{B_{p, q}^{s}}
$$

noting that

$$
\Psi_{\lambda}(t)=\lambda \int_{0}^{t}\|u\|_{\infty} d \tau .
$$

Plugging (40) into (39) then taking supremum over $[0, T)$ with respect to $t$, we have

$$
\zeta(T) \lesssim 2^{\lambda\left(1+\sup _{j} \int_{0}^{T}\left\|\Delta_{j} u\right\|_{L^{\infty}} d \tau\left(1+\log ^{+}(T \zeta(T))\right)\right)} \zeta(0) .
$$

We should point out that the previous inequality still holds if the time interval $[0, T)$ is replaced with $[T-\varepsilon, T)$. Thanks to the assumption (2) of Theorem 1, we deduce that

$$
\zeta(T) \lesssim 2^{\lambda \sup _{j} \int_{T-\varepsilon}^{T}\left\|\Delta_{j} u\right\|_{\infty} d \tau \log ^{+}(\varepsilon \zeta(T))} \zeta(T-\varepsilon) .
$$

Setting $Z(T)=\log (e+\zeta(T))$, we finally have

$$
Z(T) \lesssim \lambda \sup _{j} \int_{T-\varepsilon}^{T}\left\|\Delta_{j} \nabla u\right\|_{\infty} d \tau Z(T)+Z(T-\varepsilon) .
$$

If we choose $M=1 / C C_{0} \lambda$, condition (2) ensures the term $\lambda \sup _{j} \int_{T-\varepsilon}^{T}\left\|\Delta_{j} u\right\|_{\infty} d \tau<1 / C$ when $\varepsilon \rightarrow 0$, which implies that

$$
Z(T) \lesssim Z(T-\varepsilon)
$$

Hence, we have the $B_{p, q}^{s}$ regularity for the solution at $t=T$ and the solution can be continued after $t=T$. This completes the proof of Theorem 1 .

\section{Acknowledgments}

This work was supported by the National Natural Science Funds of China for Distinguished Young Scholar under Grant no. 50925727, the National Defense Advanced Research Project Grants nos. C1120110004, 9140A27020211DZ5102 the Key Grant Project of Chinese Ministry of Education under Grant no. 313018, and the Fundamental Research Funds for the Central Universities (2012HGCX0003).

\section{References}

[1] T. Laurent, "Local and global existence for an aggregation equation," Communications in Partial Differential Equations, vol. 32, no. 10-12, pp. 1941-1964, 2007.

[2] P. Biler and L. Brandolese, "On the parabolic-elliptic limit of the doubly parabolic Keller-Segel system modelling chemotaxis," Studia Mathematica, vol. 193, no. 3, pp. 241-261, 2009.

[3] A. L. Bertozzi and T. Laurent, "Finite-time blow-up of solutions of an aggregation equation in $\mathbb{R}^{n}$," Communications in Mathematical Physics, vol. 274, no. 3, pp. 717-735, 2007.

[4] A. L. Bertozzi, J. A. Carrillo, and T. Laurent, "Blow-up in multidimensional aggregation equations with mildly singular interaction kernels," Nonlinearity, vol. 22, no. 3, pp. 683-710, 2009.
[5] A. L. Bertozzi, T. Laurent, and J. Rosado, " $L^{p}$ theory for the multidimensional aggregation equation," Communications on Pure and Applied Mathematics, vol. 64, no. 1, pp. 45-83, 2011.

[6] D. Li and J. Rodrigo, "Finite-time singularities of an aggregation equation in $\mathbb{R}^{n}$ with fractional dissipation," Communications in Mathematical Physics, vol. 287, no. 2, pp. 687-703, 2009.

[7] D. Li and J. L. Rodrigo, "Wellposedness and regularity of solutions of an aggregation equation," Revista Matemática Iberoamericana, vol. 26, no. 1, pp. 261-294, 2010.

[8] P. Biler and G. Karch, "Blowup of solutions to generalized Keller-Segel model," Journal of Evolution Equations, vol. 10, no. 2, pp. 247-262, 2010.

[9] P. Biler, G. Karch, and P. Laurençot, "Blowup of solutions to a diffusive aggregation model," Nonlinearity, vol. 22, no. 7, pp. 1559-1568, 2009.

[10] P. Biler and G. Wu, "Two-dimensional chemotaxis models with fractional diffusion," Mathematical Methods in the Applied Sciences, vol. 32, no. 1, pp. 112-126, 2009.

[11] G. Karch and K. Suzuki, "Blow-up versus global existence of solutions to aggregation equations," Applicationes Mathematicae, vol. 38, no. 3, pp. 243-258, 2011.

[12] D. Li and J. L. Rodrigo, "Refined blowup criteria and nonsymmetric blowup of an aggregation equation," Advances in Mathematics, vol. 220, no. 6, pp. 1717-1738, 2009.

[13] P. Biler, "Existence and nonexistence of solutions for a model of gravitational interaction of particles. III," Colloquium Mathematicum, vol. 68, no. 2, pp. 229-239, 1995.

[14] M. Cannone, Q. Chen, and C. Miao, "A losing estimate for the ideal MHD equations with application to blow-up criterion," SIAM Journal on Mathematical Analysis, vol. 38, no. 6, pp. 1847$1859,2007$.

[15] Q. Chen, C. Miao, and Z. Zhang, "The Beale-Kato-Majda criterion for the 3D magneto-hydrodynamics equations," Communications in Mathematical Physics, vol. 275, no. 3, pp. 861872, 2007.

[16] Q. Chen, C. Miao, and Z. Zhang, "On the regularity criterion of weak solution for the 3D viscous magneto-hydrodynamics equations," Communications in Mathematical Physics, vol. 284, no. 3, pp. 919-930, 2008.

[17] S. Montgomery-Smith, "Finite time blow up for a NavierStokes like equation," Proceedings of the American Mathematical Society, vol. 129, no. 10, pp. 3025-3029, 2001.

[18] F. Planchon, "An extension of the Beale-Kato-Majda criterion for the Euler equations," Communications in Mathematical Physics, vol. 232, no. 2, pp. 319-326, 2003.

[19] Q. Zhang, "Local existence of solutions for an aggregation equation in Besov spaces," Colloquium Mathematicum, vol. 125, no. 2, pp. 183-192, 2011.

[20] H. Bahouri, J. Y. Chemin, and R. Danchin, Fourier Analysis and Nonlinear Partial Differential Equations, vol. 343, Springer, Heidelberg, Germany, 2011.

[21] J. M. Bony, "Calcul symbolique et propagation des singularités pour les équations aux dérivées partielles non linéaires," Annales Scientifiques de l'École Normale Supérieure, vol. 14, no. 2, pp. 209-246, 1981. 


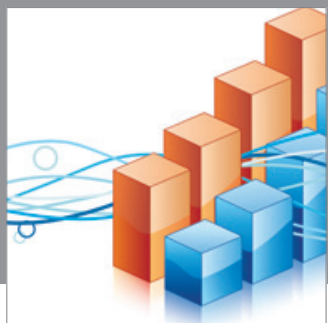

Advances in

Operations Research

mansans

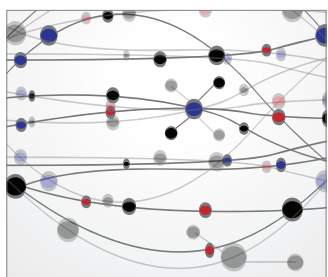

The Scientific World Journal
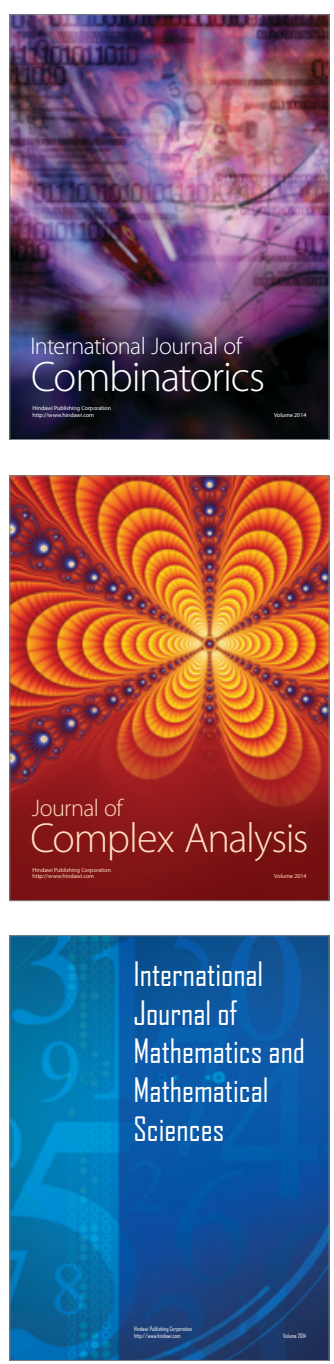
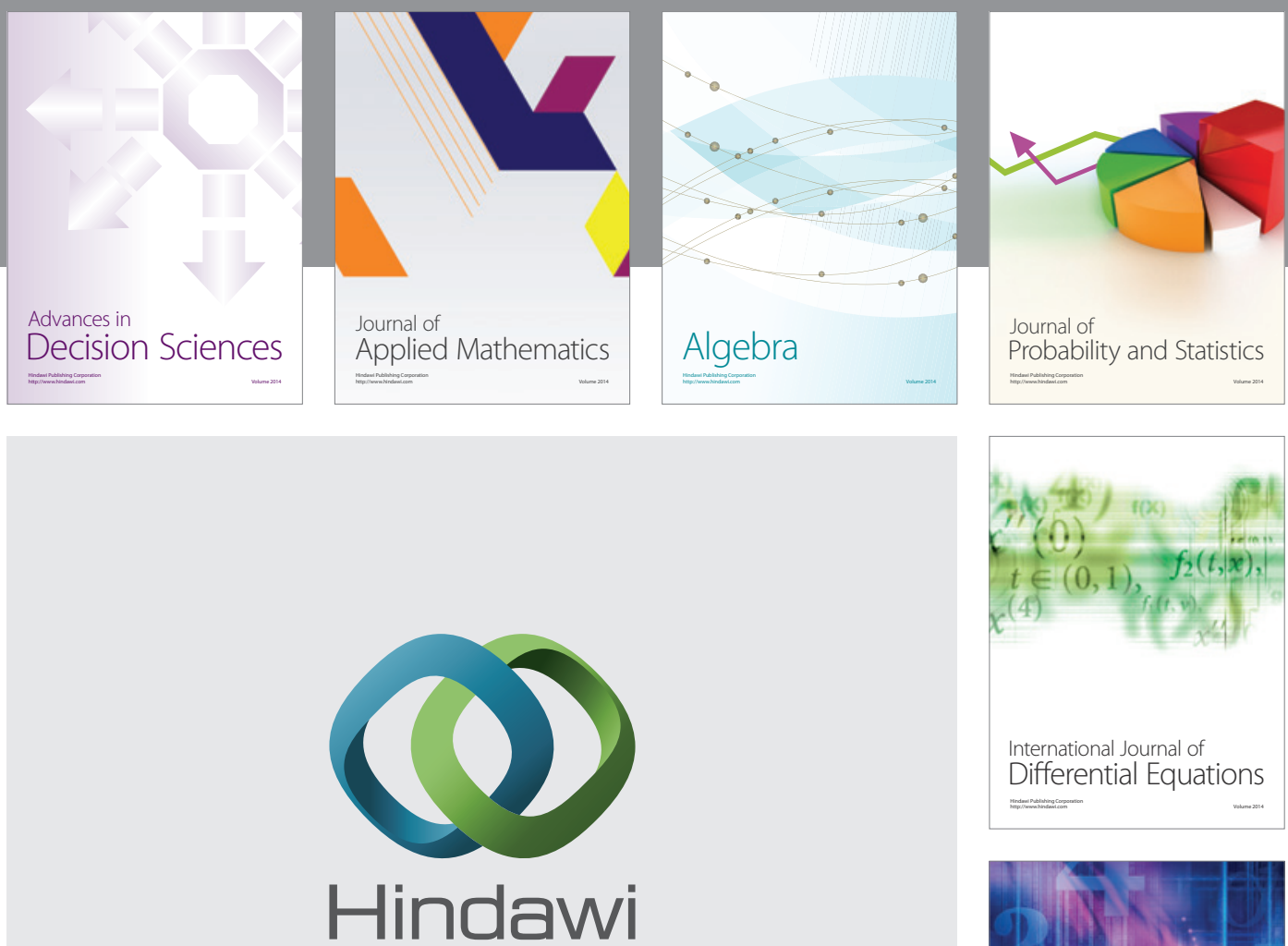

Submit your manuscripts at http://www.hindawi.com
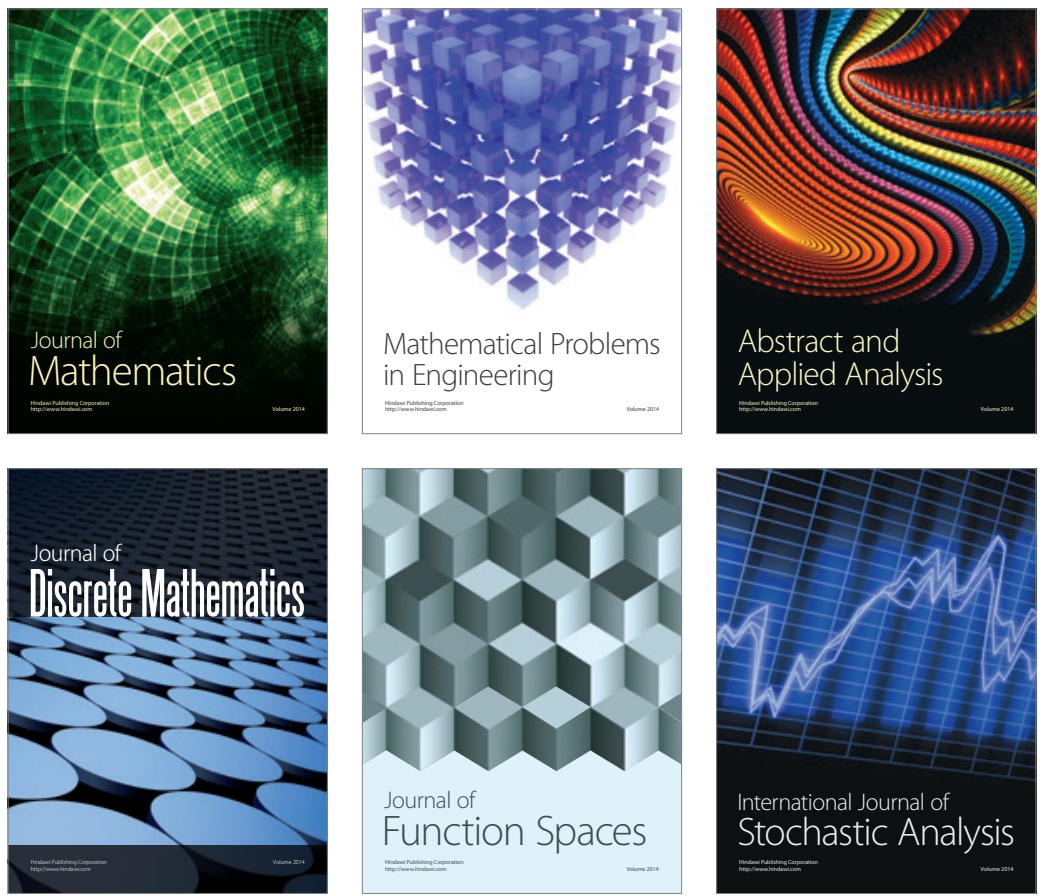

Journal of

Function Spaces

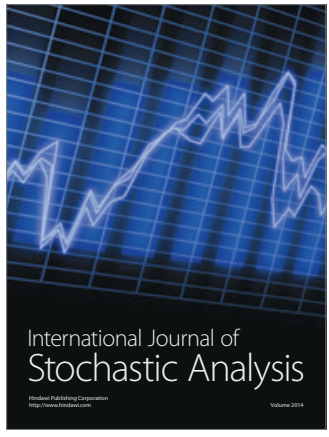

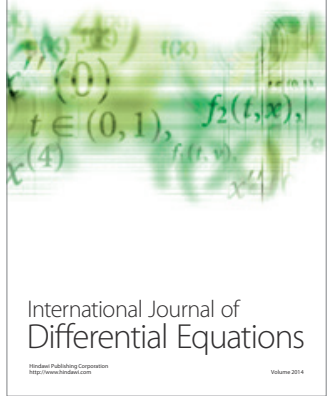
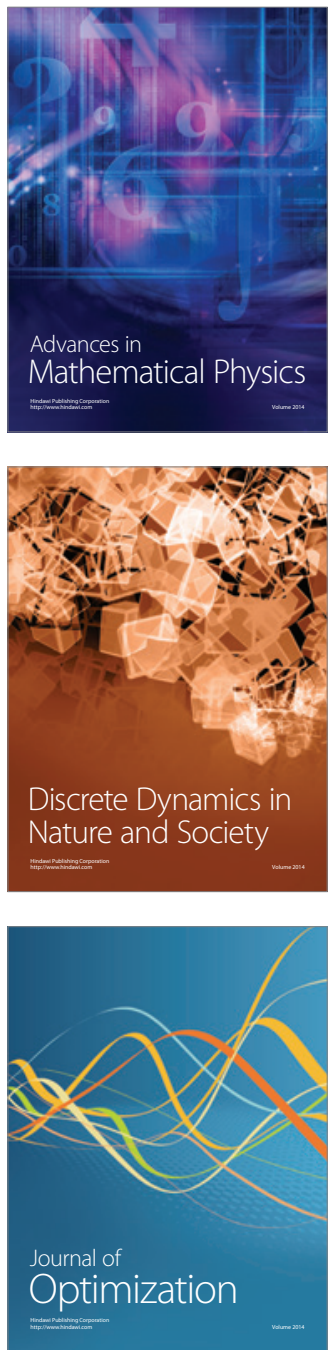